\title{
Milk from Mothers of Both Premature and Full-Term Infants Provides Better Antioxidant Protection than Does Infant Formula
}

\author{
JAMES K. FRIEL, SEAN M. MARTIN, MATTHEW LANGDON, GENE R. HERZBERG, AND \\ GARRY R. BUETTNER \\ Department of Biochemistry [J.K.F., M.L., G.R.H.], Department of Pediatrics [J.K.F.], Memorial \\ University, St. John's, Newfoundland, A1B 3X9, Canada; ESR Center, the University of Iowa, Iowa City, \\ Iowa, 52242-1101, U.S.A. [S.M.M., G.R.B.]
}

\begin{abstract}
We hypothesized that premature (PT) infants' mother's milk may provide antioxidant advantages compared with milk from mothers of full-term (FT) infants, and human milk may provide antioxidant properties not seen in infant formulas. We designed three experiments to test these hypotheses. Experiment 1 assessed resistance to oxidative stress of human milk and formulas designed for FT and PT infants. Experiment 2 determined differences in resistance to oxidative stress between milk from mothers of FT and PT infants, including analysis of catalase activity. Experiment 3 examined factors in human milk that may account for increased resistance to oxidative stress. In experiment 1 , we induced physiologic oxidative stress in human milk $(n=5)$ and formula $(n=2)$ and measured ascorbate radical using electron paramagnetic resonance. Results indicated the following: 1) during oxidative stress, ascorbate may be spared in human milk compared with formula; 2) ascorbate radical production is more intense in formula compared with human milk, with or without oxidative stress; and 3) oxygen consumption in human milk is less than that in formula, with or without oxidative stress. In experiment 2 , milk samples were collected from mothers of PT $(n=28)$ and FT $(n=17)$ infants at wk 1,2 , and 12 of lactation. No differences in oxygen consumption after oxidative stress appeared between PT and FT milk. Catalase levels in human milk increased with time. In experiment 3 , addition of catalase,
\end{abstract}

\section{ABSTRACT}

superoxide dismutase, and glutathione peroxidase to formulas ( $n=$ 4) increased resistance to oxidative stress. Denaturing endogenous enzymes did not decrease the ability of human milk to resist oxidative stress. Ferrous sulfate plus vitamin C added to human milk and formulas fortified with iron increased oxidative stress. Addition of iron chelators to formula reduced oxidative stress. In conclusion, human milk has better antioxidant protection than do formulas, perhaps because of the higher iron content of formulas. Milk from mothers of PT and FT infants has equal resistance to oxidative stress. (Pediatr Res 51: 612-618, 2002)

CAT, catalase

\section{Abbreviations}

DETAPAC, diethylenetriaminepentaacetic acid

EPR, electron paramagnetic resonance, alias ESR

ESR, electron spin resonance, alias EPR

FT, full-term

GPx, glutathione peroxidase

HM, human milk

HX, hypoxanthine

MDA, malondialdehyde

PT, premature

SOD, superoxide dismutase

XO, xanthine oxidase
HM is the ideal food during infancy (1). In addition to being the best source of nutrients, it also supplies a number of defense factors for the growing infant (2). Protection by HM resides in a complex system of host defense factors that are distinct from other mammalian milks (3). Buescher and

Received April 26, 2001; accepted December 10, 2001.

Correspondence and reprint requests: James K. Friel, Department of Biochemistry, Memorial University, St. John's, NF, A1B 3X9, Canada; e-mail: jfriel@mun.ca

Supported by Grant RR00059 from the General Clinical Research Center Program, National Center for Research Sciences, NIH; Grant 66081 from the National Institutes of Health; and Grant 146833 from the Canadian Institutes of Health Research.
McIlherhan (4) reported that human colostrum manifests antioxidant properties, being capable of spontaneous reduction of cytochrome $c$, depletion of polymorphonuclear leukocyteproduced $\mathrm{H}_{2} \mathrm{O}_{2}$, and protection of epithelial cells from polymorphonuclear leukocyte-mediated detachment. We do not know the complete list of active antioxidant components in HM. It is known, however, that scavengers of free radicals, which include $\alpha$-tocopherol, cysteine, and ascorbate, are considerably higher in HM than in cow's milk (3). In addition, GPx (EC 1.161.9), SOD (EC 1.15.1.1) (5), and CAT (EC 1.11.1.6) are also present in $\operatorname{HM}(4,5)$ to assist in the destruction of $\mathrm{H}_{2} \mathrm{O}_{2}$. 
Cow's milk is not routinely fed to human infants, but is modified into formulas that are more comparable to HM (1). These formulas have excess chain-breaking antioxidants compared with HM (1). Goldman et al. (6) state that many factors, including antioxidants, are either absent or poorly represented in cow's milk or other artificial feedings, and that the attainment of appropriate plasma levels of some antioxidants in early infancy is dependent upon the feeding of HM. Van ZoerenGrobben et al. (7) reported that premature infants who were fed HM had higher plasma peroxyl radical trapping ability in vitro than did control infants who were formula fed. Milk from mothers of PT infants is also known to vary in composition from milk from mothers of FT infants (8).

We hypothesized that milk from mothers of PT infants would have better overall antioxidant protection than milk from mothers of FT infants, and that HM has inherent antioxidant properties that infant formulas do not provide. We designed three experiments to test these hypotheses: Experiment 1 assessed resistance to oxidative stress of $\mathrm{HM}$ and artificial formulas designed for both FT and PT infants. Experiment 2 determined differences in resistance to oxidative stress between milk collected from mothers of both FT and PT infants, including a longitudinal analysis of CAT activity, one of the primary antioxidant enzymes not yet analyzed in HM. Experiment 3 examined factors in HM that may account for increased resistance to oxidative stress.

\section{MATERIALS AND METHODS}

\section{Protocol}

This study was approved by the Memorial University of Newfoundland and Health Care Corporation Ethics Committees. Informed consent was obtained from each mother.

\section{Milk Collection}

For experiment 1, samples of HM were collected from the mothers of four PT infants of varying gestational ages (25-37 wk) and one FT infant at the neonatal intensive care unit at the University of Iowa Hospital. Commercial formulas for PT infants (NeoSure, Ross Products Division, Columbus, OH, U.S.A.; Enfamil Premature Formula, Mead Johnson Nutritionals, Evansville, IN, U.S.A.) were also used. NeoSure is a specialized premature formula for infants, with $13.4 \mathrm{mg} / \mathrm{L}$ iron content, and has elevated nutrients compared with standard term formulas. Enfamil Premature Formula is a special formula for PT infants $(13.5 \mathrm{mg} / \mathrm{L}$ iron) and is for in-hospital use only (9).

For experiment 2, between milks of FT and PT infants, samples were collected from 17 mothers of FT infants and 28 mothers of PT infants at wk 1, 2, and 12 of lactation, at the Janeway Child Health Center, St. John's, NF, Canada. Wk 1 samples were collected as soon as possible after birth, between d 0 and 4; wk 2 samples were collected between d 6 and 8; and wk 12 samples between $\mathrm{d} 70$ and 74 . Each sample was collected by manual pump (Hollister, Libertyville, IL, U.S.A.) or by hand expression and immediately frozen at $-70^{\circ} \mathrm{C}$ until analysis. The volume of milk from mothers differed for each collection, and on occasion there was not enough milk available for all analyses.

For experiment 3, HM samples collected as above as well as a variety of premature formulas (from Ross Products Division and Mead Johnson Nutritionals) were evaluated to test for the contribution of known antioxidants to the ability to reveal oxidative stress.

\section{Assessing Oxidative Stress}

For both ESR studies (experiment 1) and oxygen consumption/depletion studies (experiments 1-3), HX/XO was used as a biochemical source of oxy-radicals. XO in $50 \mathrm{mM}$ phosphate buffer ( $\mathrm{pH}$ 7.4) with $\mathrm{HX}$ was added directly to milk for a final activity of $25 \mathrm{mU} / \mathrm{mL} \mathrm{XO}$ and $500 \mu \mathrm{M}$ HX. Buettner and Jurkiewicz (10) have shown that ascorbate free radical ESR signal intensity can serve as a marker for the degree of ongoing free radical oxidative stress. Therefore, for valid ESR analyses of ascorbate radical (10), vitamin C levels in the milk samples were made equal by adding vitamin $\mathrm{C}$ to $\mathrm{HM}$ samples in experiment 1 , which always contained less vitamin $\mathrm{C}$ than did formula samples (9). For oxygen consumption/depletion studies, vitamin $\mathrm{C}$ was only added to samples of HM when testing the effects of additional iron. Vitamin $\mathrm{C}$ was analyzed using the colorimetric method (hydrazine) of Omaye et al. (11).

ESR analyses were run with and without oxidative stress and until cessation of the ascorbate radical signal. Consecutive ESR spectra were taken at 48-s intervals with an ESP 300 spectrometer (Bruker Instruments, Billerica, MA, U.S.A.) at room temperature. EPR settings were $40 \mathrm{~mW}$ nominal microwave power, $0.71 \mathrm{G}$ modulation amplitude, $328 \mathrm{~ms}$ time constant, and receiver gain $5 \times 10^{5}$. Spectra were collected using a Bruker $\mathrm{TM}_{110}$ cavity and aqueous flat cell (Wilmad Glass, Buena, NJ) into which $450 \mu \mathrm{L}$ of milk sample and 50 $\mu \mathrm{L}$ of HX/XO in buffer was added. Signal height was recorded in arbitrary units.

Oxygen consumption/depletion was monitored with a YSI model 53 biologic oxygen monitor (YSI Inc., Yellow Springs, OH, U.S.A.). During oxygen depletion analysis, milk samples were measured with and without oxidative stress. Oxygen consumption/depletion was measured in three different ways: as percentage depletion from baseline, set at $100 \%$; as nmoles/ $\mathrm{mL} / \mathrm{min}$; or as total nmoles oxygen consumed over a 10 -min analysis after calibrating the instrument to read $100 \%$ consumption as $226 \mathrm{nmoles}$ oxygen $/ \mathrm{mL}$ (12). A volume of 1350 $\mu \mathrm{L}$ of HM or formula was added to the chamber with $300 \mathrm{~mL}$ of $\mathrm{HX} / \mathrm{XO}$ solution and $1350 \mu \mathrm{L}$ of phosphate buffer. For experiment 2, MDA analysis was done as a measure of lipid damage on stressed milk samples (volume $=390 \mu \mathrm{L}$ ), when available, using HPLC (13) after chloroform extraction. CAT analysis (volume $30 \mu \mathrm{L}$ ) was performed using a polarographic method (14). Not enough milk was available from mothers to complete all CAT analyses. Milk protein determination was performed using the Lowry assay and the Folin-Ciocalteu reagent with BSA $(1 \mathrm{mg} / \mathrm{mL})$ as standard. 


\section{Experiment 3: Antioxidants and Oxidants in Milks}

HM. HM, collected in bulk from a mother of a full-term infant aged $1 \mathrm{mo}$, was used for these procedures. HM was pasteurized in a hot water bath for $5 \mathrm{~min}$ at $85^{\circ} \mathrm{C}$ to denature SOD, GPx, and CAT (data not shown) and determine their contribution to antioxidant protection. HM samples were fractionated using the sucrose gradient technique (wt/vol, $10-40 \%$ sucrose). Fractions were isolated and dialyzed using Spectra/ Por membranes (Spectrum Laboratories, Rancho Dominguez, CA, U.S.A.) and individual fractions were tested for oxygen stress. HM samples were also centrifuged at 35,000 RPM for $40-45 \mathrm{~min}$ to separate casein and fat. The supernatant was put through a 30-K Amicon filter (Millipore Corporation, Bedford, MA, U.S.A.) at $6^{\circ} \mathrm{C}$ using ultracentrifugation. Nutrient Fortifier (Mead Johnson Nutritionals) was added to HM samples to test the effect on oxidative resistance. Iron $(12 \mathrm{mg} / \mathrm{L})$ and vitamin C $(80 \mathrm{mg} / \mathrm{L})$ were added to samples of $\mathrm{HM}$ to determine their effect on enhancing oxidative stress.

Formulas. Iron formulas (Similac Special Care and Similac Advance, Ross Products Division) fortified at two levels (3 and $12 \mathrm{mg} / \mathrm{L}$ ) were examined for their ability to resist oxidative stress. DETAPAC was added to iron-containing formulas to determine the effect of iron chelation on oxidative stress. CAT, GPx, and SOD at levels found in HM $(15 \mathrm{U} / \mathrm{mL}, 0.1 \mathrm{U} / \mathrm{mL}, 35$ $\mathrm{U} / \mathrm{mL}$, respectively) (5) were added to a variety of formulas for PT infants (from Ross Products Division and Mead Johnson Nutritionals) to test their ability to enhance oxidative stress.

\section{Statistical Analysis}

For experiment 1 , each formula was run once, and the PT HM sample was run twice. For experiments 2 and 3, each milk sample was analyzed six times and the mean was then calculated. MDA and CAT analyses were done in triplicate. Twotailed independent sample $t$ tests, using Levene's test for equality of variances, and repeated-measures ANOVA were performed to determine differences between PT and FT groups, with the support of the Mann-Whitney $U$ test. Between sampling times within each group, dependent sample $t$ tests were used. Group descriptives are expressed as mean \pm SEM. Pearson correlations were used to express relationship between variables. All analyses were performed with $p<0.05$ chosen as significant using SPSS (version 9.0; SPSS Inc., Chicago, IL, U.S.A.).

\section{RESULTS}

Human milk versus formula. In experiment 1, data from ESR analyses indicated that, at all times, the initial background free radical production was higher in formula than was the signal in HM samples (four FT, one PT). Once the milk samples were stressed, the rate of ascorbate radical depletion did not appear to differ from that of the formula (for four of the five HM samples). However, for one PT infant, who was the only infant to have a collection in the first week of life, there was a distinct difference in both background and slope to perdition of the ascorbate radical signal (Fig. 1). Oxygen consumption/depletion always occurred at a faster rate in

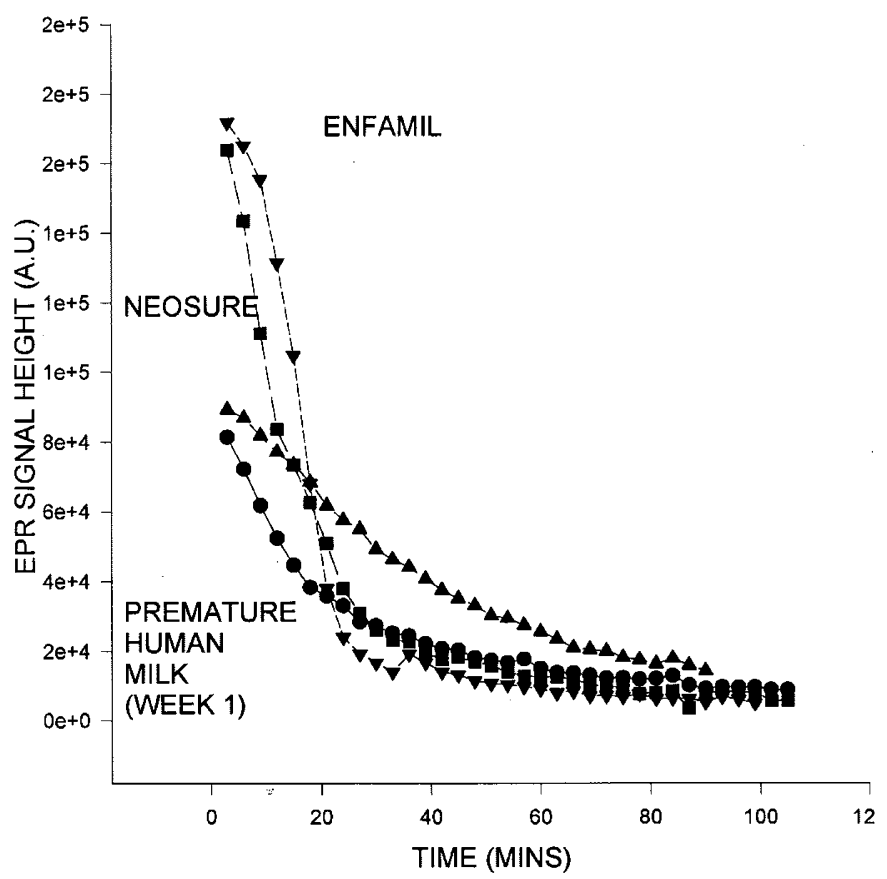

Figure 1. ESR analysis measuring ascorbate radical levels after stressing (HX/XO) two formulas for PT infants (NeoSure and Enfamil) and premature HM sample (in duplicate).

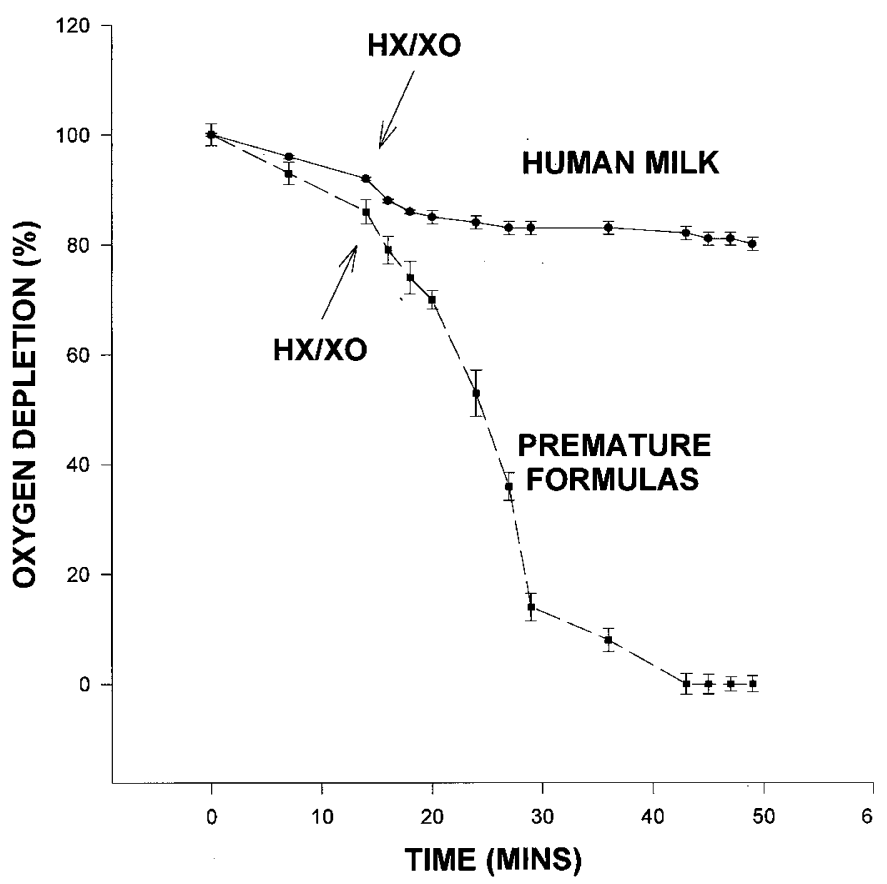

Figure 2. Average oxygen consumption/depletion for two formulas for PT infants (NeoSure and Enfamil) and HM sample with and without oxy-radicals $(n=6)$. Arrows indicate initiation of $\mathrm{HX} / \mathrm{XO}$.

formula compared with samples of HM (Fig. 2). Once oxyradicals were introduced, the rate of oxygen consumption/ depletion increased in formula at a faster rate than in HM collected at any stage of lactation.

Experiment 2: Comparison of milk from mothers of PT and FT infants. Milk samples from mothers of PT and FT infants demonstrated no significant difference between groups in oxygen consumption at any stage of lactation. There was a 
decrease in the rate of stress-induced oxygen consumption nmoles $/ \mathrm{mL} / \mathrm{min}$ after wk 1 of lactation as compared with wk 2 $(7.46 \pm 0.49, n=36 ; 4.90 \pm 0.27, n=26)$ and with wk 12 (4.40 $\pm 0.30, n=29)$ when the values for both groups are combined (Fig. 3), but no significant change between wk 2 and 12.

MDA ( $\mu \mathrm{mol} / \mathrm{L})$ results for the PT and FT groups, respectively, after addition of $\mathrm{HX} / \mathrm{XO}$ were $0.58 \pm 0.08, n=14$, and $0.72 \pm 0.11, n=8$, at wk $1 ; 0.64 \pm 0.07, n=12$, and $0.58 \pm$ $0.08, n=14$, at wk 2 ; and $0.42 \pm 0.09, n=11$, and $0.27 \pm$ $0.06, n=16$, at wk 12 . There was no significant difference observed between the groups with respect to MDA produced at either wk 1,2, or 12 of lactation. There was no initial decrease in MDA levels from wk 1 to wk 2 when values were combined, but a significant decrease was observed from wk $1(0.60 \pm$ $0.31, n=22)$ and wk $2(0.60 \pm 0.28, n=26)$ to wk 12 of lactation $(0.32 \pm 0.26, n=27)$.

CAT values for the specific activity (in $\mathrm{U} / \mathrm{mg}$ protein: 0.50 $\pm 0.08, n=12$, and $0.43 \pm 0.05, n=8$, for wk $1 ; 0.72 \pm 0.10$, $n=11$, and $0.82 \pm 0.13, n=14$, for wk 2 ; and $0.97 \pm 0.21$, $n=13$, and $0.84 \pm 0.12, n=16$, for wk 12 of lactation for PT and FT milk samples, respectively) did not differ. When values were combined, there was an increase in specific activity (U/mg protein) over time from wk 1 to wk $2(0.47 \pm 0.05, n$ $=20 ; 0.78 \pm 0.08, n=25)$ but no change between wk 2 and $12(0.90 \pm 0.61, n=29)$. Protein content of milk samples was $24.2 \pm 9.5 \mathrm{mg} / \mathrm{mL}(n=20)$ at $\mathrm{wk} 1,19.7 \pm 3 \mathrm{mg} / \mathrm{mL}(n=25)$ at wk 2 , and $16.2 \pm 5 \mathrm{mg} / \mathrm{mL}(n=29)$ at wk 12 . Protein content differed between PT $(18 \pm 6 \mathrm{mg} / \mathrm{mL})$ and FT $(14.2 \pm$ $2.1 \mathrm{mg} / \mathrm{mL}$ ) at wk 12. Protein content dropped significantly at each sampling time. No difference between groups was observed when data were expressed as units per milliliter of milk

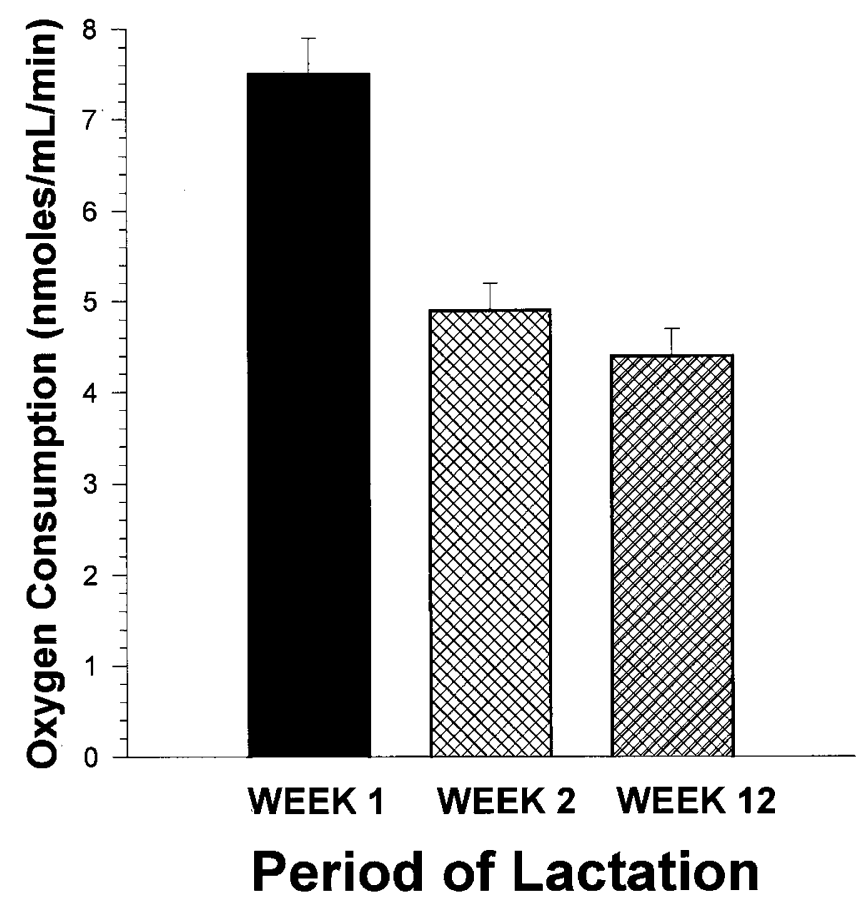

Figure 3. Oxygen consumption (nmoles $/ \mathrm{mL} / \mathrm{min}$ ) after oxidative stress (HX/ $\mathrm{XO}$ ) at weeks 1,2 , and 12 in milk of all study mothers (mean $\pm \mathrm{SEM}, p<$ $0.05)$.

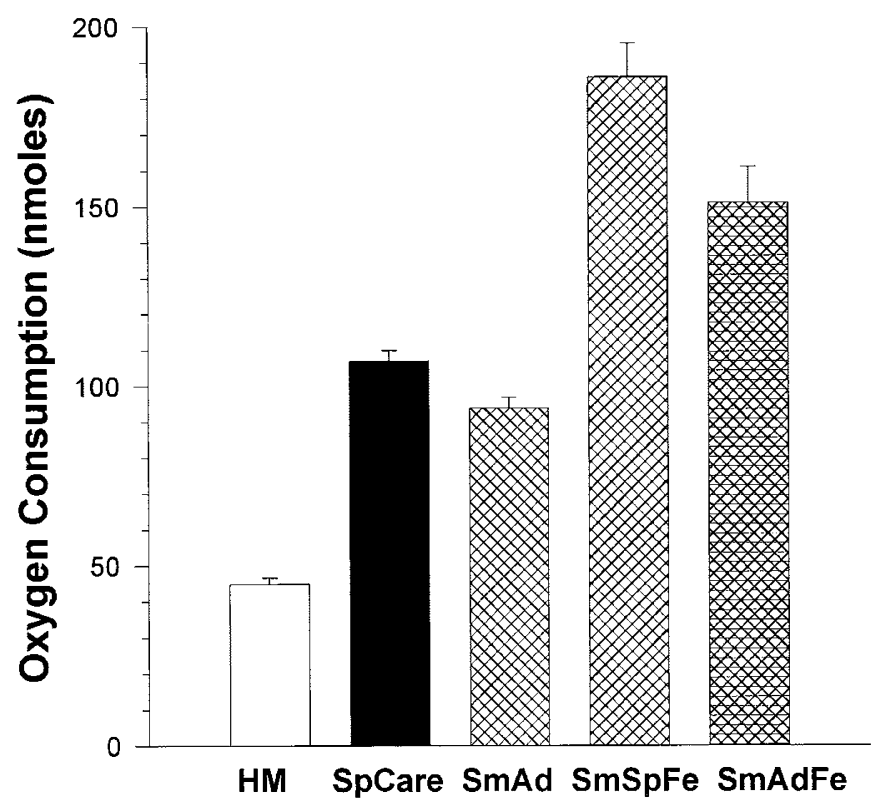

\section{Formula Group}

Figure 4. Comparison of oxygen consumption (total nmoles $/ \mathrm{mL} / 10 \mathrm{~min}$ ) in $\mathrm{HM}$ and infant formulas after oxidative stress $(\mathrm{HX} / \mathrm{XO})$ (mean $\pm \mathrm{SEM} ; p<$ 0.05), including Similac Special Care with (SmSpFe) and without (SpCare) iron and Similac Advance with $(S m A d F e)$ and without $(S m A d)$ iron.

at any sampling time. When values for CAT were combined, there was an increase in activity over time from wk 1 (10.1 \pm $6, n=36)$ to wk $2(14.4 \pm 8.4, n=28)$ but not to wk $12(14.4$ $\pm 10, n=31)$. We found no difference in CAT activity after freezing, storage, or thawing before analysis. Commercial formula had no CAT activity.

A significant $\left(r^{2}=0.443\right)$ positive correlation existed between the level of MDA observed in both groups combined at wk 2 of lactation and the level of oxygen consumption observed during wk 1. A negative correlation $\left(r^{2}=-0.30\right)$ also existed between the overall level of oxygen consumption observed and the specific activity of CAT for values of wk 1,2, and 12 combined. No correlation was found between the level of MDA and specific activity of CAT at any stage of lactation or with all weeks combined.

Experiment 3: Factors in HM that may account for increased resistance to oxidative stress. A difference was found in total oxygen consumption (nmoles) between the HM [44.8 \pm 1.7 (mean \pm SEM) ] and both the low-iron $(1.5-3 \mathrm{mg} / \mathrm{L}$ iron: $99 \pm 3.1)$ and standard-iron $(12-14 \mathrm{mg} / \mathrm{L} ; 169 \pm 9.5)$ formulas after the addition of the free radical generator (Fig. 4). These results were consistent for all samples of HM examined at any stage of lactation. Formulas with standard levels of iron exhibited nearly a 2 -fold increase in oxygen consumption compared with the same formula with low iron levels. Addition of DETAPAC significantly reduced the oxidative stress seen in iron-fortified formulas (data not shown).

Both low- and standard iron formulas were tested for their ability to resist oxidative damage with and without the addition of CAT, SOD, and GPx. Addition of these enzymes signifi- 


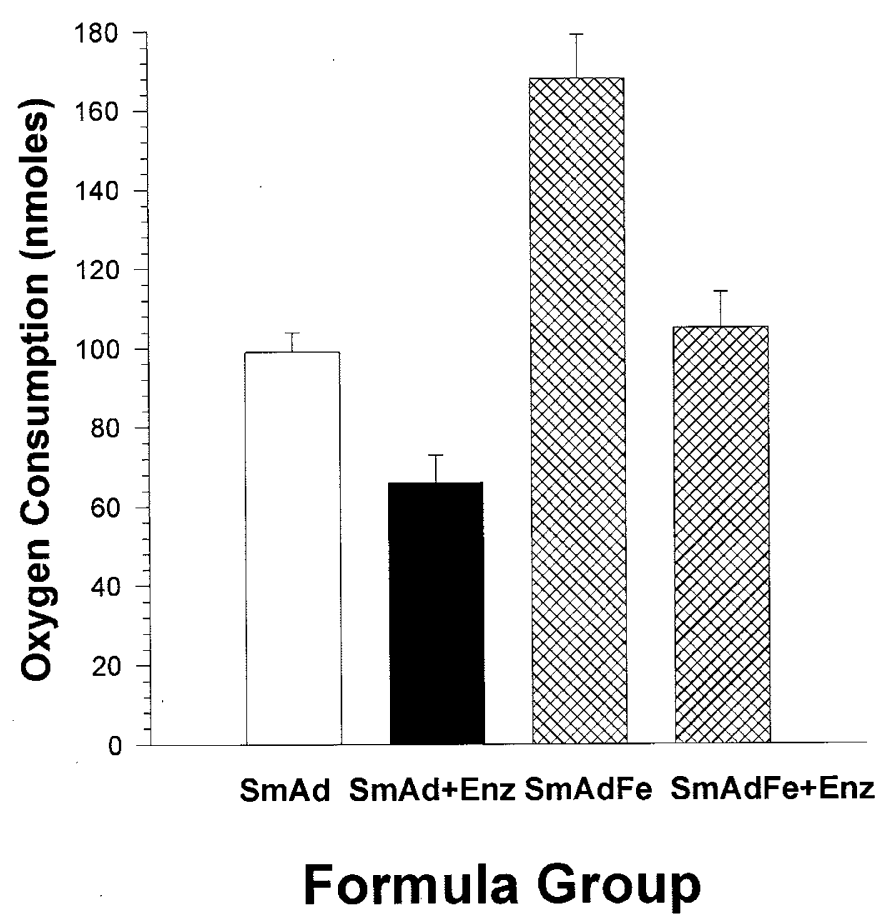

Figure 5. Oxygen consumption (total $\mathrm{nmoles} / \mathrm{mL} / 10 \mathrm{~min}$ ) after stress (HX/ $\mathrm{XO})$ in low $(3 \mathrm{mg} / \mathrm{L})$ and normal $(12 \mathrm{mg} / \mathrm{L})$ iron-fortified formulas after addition of the enzymes CAT, SOD, and GPx (mean \pm SEM, $p<0.05$ ). $S m A d$, Similac Advance; $S m A d+E n z$, Similac Advance plus enzymes; SmAdFe, Similac Advance with iron; SmAdFe+Enz, Similac Advance with iron plus enzymes.

cantly increased antioxidant capacity by decreasing the total oxygen consumption (nmoles) of the low- and standard-iron formulas to $66 \pm 6.5$ and $105 \pm 8.7$, respectively (Fig. 5).

Addition of iron and vitamin $\mathrm{C}$ to $\mathrm{HM}$ resulted in a 2-fold increase in oxygen consumption ( 81.5 nmoles) upon addition of $\mathrm{HX} / \mathrm{XO}$ in the samples containing iron compared with $\mathrm{HM}$ controls. Samples with only vitamin C added to HM were not different from controls (49.3 nmoles) (Fig. 6). Neither the addition of $\mathrm{HM}$ fortifier nor different fractions by density or molecular weight affected the antioxidant properties of HM samples. There was no difference between HM milk samples before and after heat treatment with respect to oxygen consumption during oxidative stress (data not shown).

\section{DISCUSSION}

Oxidation of human milk and premature formula using ESR analysis (experiment 1). Of the five HM samples examined in experiment 1 , in comparison with specialized formulas for PT infants, not all HM samples were able to maintain better protection against oxy-radical attack when measured to perdition of the ascorbate radical by ESR. However, all HM samples displayed a lower initial rise in the ascorbate signal, suggesting a buffering effect at the commencement of free radical attack. Further, one particular sample (Fig. 1) showed both a slower initial response and longer duration than did the artificial formulas. Why this particular sample of milk from a mother of a premature infant had better antioxidant properties was uncertain. Although we were unable to determine the exact time

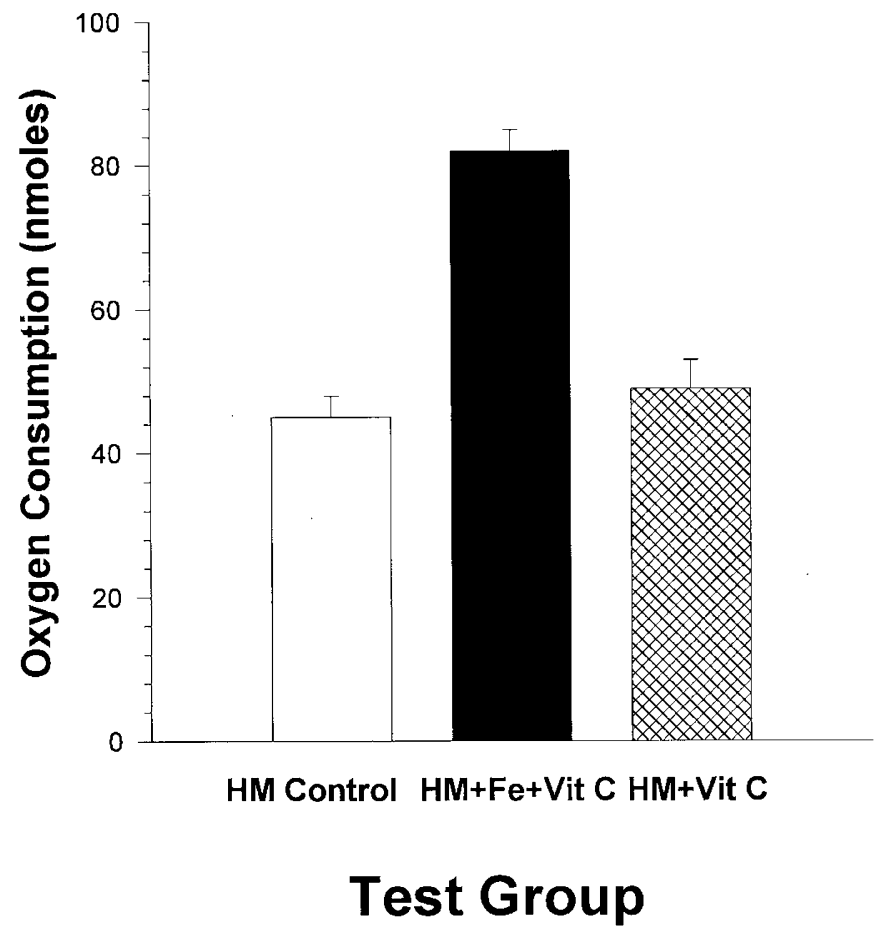

Figure 6. Effect of addition of formula-equivalent levels of iron $(\mathrm{Fe})$ and vitamin $\mathrm{C}$ (Vit $C$ ) on oxygen consumption (total $\mathrm{nmoles} / \mathrm{mL} / 10 \mathrm{~min}$ ) after oxidative stress $(\mathrm{HX} / \mathrm{XO})$ ). FT HM collected at 1 mo of age (mean $\pm \mathrm{SEM}, p$ $<0.05)$.

of the sample collection, nurses' notes indicated that the sample was collected in the first week after birth. The results of this particular sample lead us to hypothesize that PT HM would have more resistance than FT HM to oxidative attack, particularly during wk 1 . Therefore, we initiated collection of samples over $12 \mathrm{wk}$ from a total of 45 mothers. (17 FT, $28 \mathrm{PT}$.)

Comparison of milk from mothers of FT and PT infants. Buescher and Mcllherhan (4) suggested that colostrum has heterogeneous antioxidant capability that may aid the immature antioxidant defense system of the premature infant (15). Saugstad (16) and Sullivan (17) have suggested that the critical diseases of prematurity are due to an imbalance of antioxidant defense and exposure to free radicals brought about by hypoxia/reperfusion injury. This attack occurs shortly after birth, and the exposure of PT infants to excess free radicals places them at risk for intraventricular hemorrhage, bronchopulmonary dysplasia, retinopathy of prematurity, and necrotizing enterocolitis. Because of our initial experiments and because PT infants seem to have underdeveloped antioxidant protection (15), we hypothesized that HM of the PT infant would compensate for this underdevelopment, as it does for the compensation of essential nutrients (8). Therefore, HM samples from wk 1, 2, and 12 of lactation were collected from mothers of PT and FT infants for analysis. The first 2 wk of lactation were chosen because it is during this time that oxidative damage is most severe, and any antioxidant protection that can be provided would be most beneficial. Week 12 was chosen to examine how HM antioxidant properties vary throughout the duration of lactation and to represent "mature milk." From our results, preterm HM samples demonstrated the same antioxi- 
dant characteristics as did samples from the FT group. There was the same amount of lipid damage and CAT-specific activity at all stages of lactation, clearly illustrating that the PT milk samples do not exhibit enhanced antioxidant properties over and above those seen in FT milk samples. However, although the PT samples did not contain more antioxidant capacity, they were as effective in resisting oxidative stress and preventing the formation of MDA as were those in the FT group compared with formulas. To our knowledge, there are no other studies comparing the antioxidant capacity of milk from mothers of FT and PT infants, even though they are known to differ in nutrient composition $(8,18)$. Coping with excess oxygen exposure in PT infants is a relatively recent concern, as most PT infants did not survive 40 years ago. Technology has advanced more quickly than the biologic alteration of HM in adapting to early birth.

Heyndrickx (19) was the first to report an approximation of CAT activity in HM to be about 10 times greater than the level determined in cow's milk. We report here longitudinal values for the specific activity of CAT in milk from mothers of both PT and FT infants. Both groups demonstrated the same activity at wk 1,2, and 12 of lactation, illustrating that milk from mothers of low-birth-weight infants is not compromised with respect to this antioxidant enzyme. The specific activity of the enzyme was also seen to increase with the length of lactation to a near 2-fold difference between wk 1 and wk 12 because of the decline in protein content. When expressed per milliliter of milk, there was also a change over time between wk 1 and 2 . This was the opposite of that which we expected, because it would seem appropriate for the mother to have an increased expression of the gene for the CAT enzyme during the period when the infant would be at most oxidative risk, and could benefit from extra antioxidant protection. Heyndrickx (19) found a trend to decrease in activity between colostrum (3-5 d) levels and normal milk (7-32 d), however, samples were not collected from the same mother.

$\boldsymbol{H M}$ versus infant formula. The potential for HM to directly affect oxygen-induced tissue injury in the newborn is supported by experimental studies in animals. Neonatal rat intestine will peroxidize HM, supporting the presence of reactive oxygen species in the gut (20). The release of oxygen radicals by the intestine has been implicated in the pathology of necrotizing enterocolitis (21). Early feeding of HM reduces the incidence of necrotizing enterocolitis in the premature infant (22). Breast-fed premature infants have a lower incidence of retinopathy of prematurity (23). Pitt et al. (24) and Lucas and Cole (22) have suggested that the lower incidence of necrotizing enterocolitis in breast-fed babies may be due to some property of HM. Furthermore, common neonatal infections have been shown to be lower in infants receiving milk from mothers of PT infants than in infants receiving the prescribed nursery formula (25). Our results indicating that HM is less susceptible to oxidative stress than are specialized premature formulas - as seen by some $(26,27)$, but not others $(20)$, using a variety of model systems - adds support to the idea that it may be the antioxidant properties of $\mathrm{HM}$ that afford a part of this protection.
Formulas for PT infants are heavily fortified with vitamins $\mathrm{E}, \mathrm{A}$, and $\mathrm{C}$ (the small-molecule antioxidants), which are found in higher concentration in formulas than in HM (9). This implies that the protective effect of milk is due to factors other than these antioxidants and may involve milk enzymes. We (5) and others have reported on the presence of GPx and SOD in HM. We hypothesized that the antioxidant enzymes CAT, SOD, and GPx present in HM would play a role in providing the HM with its antioxidant capability in vitro. However, upon pasteurization of the HM and inactivation of these enzymes, its antioxidant properties were not compromised upon oxidative stress, suggesting that HM exhibits alternate, and equally effective, means of dealing with excess oxidative stress and does not depend solely upon the known antioxidant enzymes. Buescher et al. (28), suggest that colostrum antioxidant activity is heterogeneous, which may explain our inability to isolate one specific compound accounting for milk antioxidant protection. This also makes sense if we consider that HM is exposed to an acid and an enzymatic environment before it moves to the small intestine to help protect against necrotizing enterocolitis. Nonetheless, the antioxidant enzymes CAT, SOD, and GPx, when added together to formula, were shown to provide increased protection against oxidative stress and lipid damage, likely working with other systems in the HM. This suggests that the addition of these protective agents to formulas may help reduce the harmful effects of excess oxidative stress and inflammatory-induced reactive oxygen species, resulting in reduced symptoms, faster recovery, and normal development, as has been shown previously with SOD given intramuscularly (29). Consistent with these results is the report of Van ZoerenGrobben et al. (7), who found that healthy breast-fed PT infants had higher antioxidant capacity in plasma compared with formula-fed infants. The results of Marshall and Roberts (20) showing that the survival of newborn rat pups exposed to $>95 \%$ oxygen declined when fed with HM compared with formula feeding are less easy to comprehend.

Iron as a free radical generator in milk. Vitamin $\mathrm{C}$ and iron are found in high quantities in most neonatal formulas, and potentially at levels that allow for a source of free iron that is capable of initiating free radical reactions $(30,31)$. When ferrous iron reduces $\mathrm{H}_{2} \mathrm{O}_{2}$ to produce ${ }^{\bullet} \mathrm{OH}$, it becomes ferric iron. In the presence of vitamin $\mathrm{C}$, the iron is converted back to its ferrous state and is again available to begin another cycle of ${ }^{\bullet} \mathrm{OH}$ formation if it is not sequestered by iron storage or transfer proteins (32). In the present study, when iron and vitamin $\mathrm{C}$ but not vitamin $\mathrm{C}$ alone were added to $\mathrm{HM}$ at equivalent levels found in formula, there was a greater increase of oxygen consumption, indicating that the iron was initiating free radical reactions. The increase in oxygen consumption in HM thus treated was not as great as levels seen in iron-fortified formulas, a finding reported by others (26). This confirms that iron content per se, does not account for all oxidative stress seen in formulas. It is possible that increased polyunsaturated fatty acid content seen in premature formulas (9) may provide more substrate for free radical attack.

In our study, formula with $12-14 \mathrm{mg} / \mathrm{L}$ iron demonstrated a 2 -fold increase in the amount of oxygen consumed compared with the same formula containing low amounts of iron (1.5-3.0 
$\mathrm{mg} / \mathrm{L})$. A decreased production of free radicals in these formulas was found upon the addition of the iron binder DETAPAC. A correlation of iron content in infant nutrient preparations with peroxidation products was reported by Marshall and Roberts (20). The addition of both CAT and desferrioxamine has previously been shown to inhibit radical formation in formulas (26), supporting a role for iron chemistry. It may be that lactoferrin, an iron binder present in HM, may function not only as an inhibitor of bacterial growth but may act to prevent free radical reactions (33).

In summary, these data support further advantages for HM in protecting against oxidative stress in the newborn premature infant. The precise mechanisms for this protection are not yet clear, however, this ability appears to be heterogeneous.

Acknowledgments. The authors thank Dr. Freya Schaefer, Dr. Steven Qian, Brett R. Wagner, Allison MacDonald, and Claude Mercer for their technical assistance, as well as all the mothers who participated and Drs. Wayne Andrews and Khalid Aziz for their critical advice. We also thank the nurses at the Iowa Clinical Research Center and Jack Widness for their assistance.

\section{REFERENCES}

1. Anderson GH 1985 Human milk feeding. Pediatr Clin North Am 32:335-353

2. Goldman AS, Goldblum RM 1995 Defence agents in human milk. In: Jensen R (ed) Handbook of Milk Composition. Academic Press, San Diego, pp 727-745

3. Goldman AS, Thorpe LW, Goldblum RM, Hanson KA 1986 Anti-inflammatory properties of human milk. Acta Paediatr Scand 75:689-695

4. Buescher ES, McIlherhan SM 1988 Antioxidant properties of human colostrum. Pediatr Res 24:14-19

5. L'Abbe M, Friel JK 2000 Superoxide dismutase and glutathione peroxidase content of human milk from mothers of premature and full-term infants during the first 3 months of lactation. J Pediatr Gastroenterol Nutr 31:270-274

6. Goldman AS, Goldblum RM, Hanson KA 1990 Anti-inflammatory systems in human milk. Adv Exp Med Biol 262:69-76

7. Van Zoeren-Grobben D, Lindeman JH, Houdkamp E, Brand R, Schrijver J, Berger HM 1994 Postnatal changes in plasma chain-breaking antioxidants in healthy preterm infants fed formula and/or human milk. Am J Clin Nutr 60:900-906

8. Lonnerdal B, Atkinson SA 1995 Nitrogenous components of milk. In: Jensen R (ed) Handbook of Milk Composition. San Diego, Academic Press, pp 351-368

9. Abbott Laboratories 1999 Composition of Feedings for Infants and Young Children. Ross Products Division, Columbus, OH, pp 1-17

10. Buettner GR, Jurkiewicz BA 1993 Ascorbate free radical as a marker of oxidative stress: an EPR study. Free Radic Biol Med 14:49-55

11. Omaye ST, Turnbull JD, Sauberlich HE 1979 Selected methods for the determination of ascorbic acid in animal cells, tissues and fluids. Methods Enzymol 62:3-11
12. Altman PL, Gibson Jr JF, Wang CC 1958 Handbook of Respiration. Wright Air Development Center, Fairborn, Ohio

13. Lepage G, Munoz G, Champagne J, Roy CC 1991 Preparative steps necessary for the accurate measurement of malondialdehyde by high-performance liquid chromatography. Anal Biochem 197:277-283

14. Hirvi Y, Griffiths MW 1998 Milk catalase activity as an indicator of thermization treatments used in the manufacture of cheddar cheese. J Dairy Sci 81:338-345

15. Inder TE, Graham P, Sanderson K, Taylor BJ 1994 Lipid peroxidation as a measure of oxygen free radical damage in the very low birthweight infant. Arch Dis Child 70:F107-F111

16. Saugstad OD 1988 Hypoxanthine as an indicator of hypoxia: its role in health and disease through free radical production. Pediatr Res 23:143-150

17. Sullivan JL 1988 Iron, plasma antioxidants and the "oxygen radical disease of prematurity." Am J Dis Child 142:1341-1344

18. Atkinson SA 1995 Effect of gestational stage at delivery on human milk components. In: Jensen R (ed) Handbook of Milk Composition. San Diego, Academic Press, pp $222-234$

19. Heyndrickx GV 1963 Further investigations on the enzymes in human milk. Pediatrics June 1963;31:1019-1023

20. Marshall TA, Roberts RJ 1990 In vitro and in vivo assessment of lipid peroxidation of infant nutrient preparations; effect of nutrition on oxygen toxicity. J Am Coll Nutr 9:190-199

21. Brown EG, Sweet AY 1982 Neonatal necrotizing enterocolitis. Pediatr Clin North Am 29:1149-1170

22. Lucas A, Cole TJ 1990 Breast milk and necrotizing enterocolitis. Lancet 336:15191523

23. Cunningham AS 1987 Breast feeding, antioxidants and the retinopathy of prematurity. Am J Obstet Gynecol 156:1040-1041

24. Pitt J, Barlow B, Heird WC 1977 Protection against experimental necrotizing enterocolitis by maternal milks. 1. Role of milk leucocytes. Pediatr Res 11:906-909

25. Narayanan I, Prakash K, Gujral VV 1981 The value of human milk in the prevention of infection in the high-risk low-birth-weight infant. J Pediatr 99:496-498

26. Almaas R, Rootwelt T, Oyasaeter S, Saugstadt D 1997 Ascorbic acid enhances hydroxyl radical formation in iron-fortified infant cereals and infant formulas. Eur J Pediatr 156:488-492

27. Van Zoeren-Grobben D, Moison RM, Ester WM, Berger HM 1993 Lipid peroxidation in human milk and infant formula: effect of storage, tube feeding and exposure to phototherapy. Acta Paediatr Scand 82:645-649

28. Buescher ES, McIlherhan SM, Frenck RW 1989 Further characterization of human cholesterol antioxidants: identification of an ascorbate like element as an antioxidant component and demonstration of antioxidant heterogeneity. Pediatr Res 25:266-270

29. Rosenfeld W, Evans H, Concepcion L, Jhaveri R, Schaeffer H, Friedman A 1984 Prevention of bronchopulmonary displasia by administration of bovine superoxide dismutase in preterm infants with respiratory distress syndrome. J Pediatr 105:781785

30. Herbert V, Shaw S, Jayatilleke E, Stopler-Kasdan T 1994 Most free radical injury is iron related: it is promoted by iron, hemin, holoferritin, and vitamin $\mathrm{C}$, and inhibited by desferoxamine and apoferritin. Stem Cells 12:289-303

31. Qian SY, Buettner GR 1999 Iron and dioxygen chemistry is an important route to initiation of biological free radical oxidations: An electron paramagnetic resonance spin trapping study. Free Radic Biol Med 26:1447-1456

32. Buettner GR, Jurkiewicz BA 1996 Catalytic metals, ascorbate, and free radicals: combinations to avoid. Radiat Res 145:532-541

33. Raghuveer T, McGuire EM, Wagner BA, Buettner GR, Widness JA 2001 Lactoferrin (Lf) supplementation can reduce iron-induced oxidative stress (abstract 1675). In: Program of the Pediatric Academic Societies and American Academy of Pediatrics Joint Meeting, Baltimore, MD, U.S.A., April 28-May 1, 2001. Lippincott Williams \& Wilkins, Baltimore, vol. 49, p 293A 